\section{Organic Mass Spectrometry in Art and Archaeology}

by Maria Perla Colombini and Francesca Modugno (eds): Wiley, Chichester, UK 2009, pp xiii+494, ISBN 978-0-470-51703-1.

Price: GBP 120.00, EUR 138.00

The identification of amorphous organic substances associated with archaeological materials and works of art has long fascinated and motivated the analytical chemist. Prior to the development of instrumental analytical techniques, chemists applied myriad tests to determine the chemical and physical properties of these intractable samples. Their efforts served to highlight the challenges of working with trace amounts of organic matter, often mixed together in varying quantities, and subject to complex sequences of degradation and contamination. Applications of mass spectrometry have revolutionised the study of such samples and this volume is a very useful addition to a relatively small but growing field of enquiry. And it is not a scientific cul-de-sac; one of the contributors to this volume (Evershed) has just been elected a Fellow of the Royal Society in the UK, in part for his groundbreaking research into molecular archaeology.

The editors, both from the University of Pisa, divide the volume into five parts.
Part I (introduction) contains background chapters on the most common molecular compound classes encountered in art and archaeology and a survey of mass spectrometric techniques. Part II (direct mass spectrometric analysis) contains four chapters. Part III (gas chromatography/mass spectrometry) has six chapters covering lipids, resins and proteinaceous binders and includes pyrolysis-GC-MS applications. Part IV (liquid chromatography/mass spectrometry) consists of only one chapter on the analysis of natural dyestuffs. Finally, Part V (other MS-based techniques) explores the considerable advances in the isotopic analysis of single compounds, novel applications of TOF-SIMS to 'chemical imaging' of multiple organic species and AMS dating.

The case studies are slightly biased towards art historical applications but, as an archaeological scientist, this does not detract as these allow similarities and differences to be drawn to one's own field. The book is excellent at covering the molecular 'common ground' between art and archaeology. Thus the reader will find considerable detail on the investigation of lipids, terpenoid resins, protein binders, waxes and dyes. The isotope analysis of single compounds features in one comprehensive chapter with numerous archaeological applications but readers will have to look elsewhere for applications of bulk isotope analysis-an approach widely used in archaeology for palaeodietary reconstruction but with wider applications to cultural heritage. Similarly, there are few applications of LC-MS/MS analysis - an approach becoming more widely used in archaeology for the analysis and identification of more exotic substances such as traces of wine in ancient ceramic containers.

What impresses me most about this volume is not so much the diversity of mass spectrometric approaches that can be deployed in the study of cultural heritage but the considerable advances that have been made in characterising organic substances and in documenting the effects of heat, light, oxygen and microorganisms on the long-term transformation of organic molecules. Most of the chapters blend succinct and well-organized technical reviews with relevant and up-to-date applications, including numerous illustrations and references. Unusually, the referencing style varies from chapter to chapter. There is some repetition in the volume and not all of the chapters could be labelled cutting edge. Minor criticisms aside, this is a really useful sourcebook and should be consulted by everyone working in the field.

C. Heron 